\title{
RESUMEN
}

En la actualidad más de un tercio de la población mundial utiliza el Internet para comunicarse. Los Sitios de Redes Sociales (SNSs), son una de las plataformas que facilita el intercambio de información. La mayoría de las empresas que crean perfiles en SNSs, lo hacen para captar nuevos clientes y fidelizar a los actuales. Sin embargo, en la región de Coquimbo, actualmente no existen investigaciones sobre la efectividad de la influencia que tienen estos sitios en la venta de productos y servicios. Este artículo científico discute un caso de estudio de la red Facebook de dos empresas de los principales sectores económicos representativos de la región, "Callegari Automotriz" (comercio) y "Turismo Delfines" (turismo). Luego de la realización de entrevistas y encuestas y un análisis estadísticos de los resultados, se determina que las empresas de la región de Coquimbo no utilizan eficientemente las Fan Pages de Facebook, perdiendo oportunidades de venta de productos y servicios.

Palabras Claves: sitios de redes sociales, eWOM, facebook.

\section{CASO DE ESTUDIO SOBRE LA EFECTIVIDAD EN LA UTILIZACIÓN DE FACEBOOK EN LA VENTA DE PRODUCTOS Y SERVICIOS EN EMPRESAS DE LA REGIÓN DE COQUIMBO.}

María José Gálvez Gutiérrez $/$ / Paullette Ailyn Salinas Cruzat 2 /Camilo Andrés San Martín Jeraldo $_{3} /$ Paul Leger $_{4} /$ Carmen Hidalgo-Alcázar ${ }_{5}$

Fecha de recepción: 05 de Septiembre de 2020

Fecha de aceptación: 02 de Noviembre de 2020

DOI: https://doi.org/10.22370/riace.2020.9.1.2597

1 Corresponding Author. Escuela de Ciencias Empresariales, Universidad Católica del Norte. mariajose_ gg@hotmail.com

2 Escuela de Ciencias Empresariales, Universidad Católica del Norte. psalinascruzat@gmail.com 3 Escuela de Ciencias Empresariales, Universidad Católica del Norte. csanmartin90@gmail.com 4 Escuela de ingeniería, Universidad Católica del Norte. pleger@ucn.cl

5 Facultad de Ciencias Económicas y Empresariales, Universidad de León (España). mhida@unileon.es 


\title{
CASE STUDY ABOUT EFFECTIVENESS IN THE USE OF FACEBOOK FOR PRODUCT AND SERVICES SELLINGS IN COMPANIES FROM COQUIMBO REGION
}

\begin{abstract}
Currently more than one third of the world's population uses the Internet to communicate. Social Networking Wepages (SNW) are a type of platforms that facilitates the information exchange. Most of the companies that create profiles on SNWs do so to attract new customers and retain the older ones. However, in the Coquimbo Region of Chile, there is no research about the effectiveness of the influence these wepages would have on products and services sellings. This research discusses a case study of the Facebook network of two companies from the main economic sectors in the region, "Callegari Automotriz" (commerce) and "Turismo Delfines" (tourism). After conducting interviews and surveys and a statistical analysis of the results, it is determined that companies in the Coquimbo Region do not use Facebook Fan Pages efficiently, losing opportunities to sell products and services.
\end{abstract}

Keywords: sitios de redes sociales, eWOM, facebook. 


\section{INTRODUCCIÓN}

Actualmente la tecnología ha revolucionado la forma de comunicación, siendo internet la responsable de ser la principal red informativa del mundo, ya que, a través de los modernos dispositivos tecnológicos y plataformas digitales se han facilitado la comunicación entre las personas, naciendo a su alero una gran cantidad de Sitios de Redes Sociales o SNSs con el objetivo de interactuar, compartir contenidos y crear comunidades sobre intereses similares (Hanna, R., Rohm, A., \& Crittenden, V.L., 2011).

Existen una gran cantidad de tipos de SNSs con diversas características, de los cuales los más utilizados en Chile son Facebook, Twitter, YouTube, Google +, entre otros (Burson-Marsteller, 2010).

Los SNSs se han transformado en una herramienta fundamental para promocionar los productos o servicios de una empresa, ya que las características de estos sitios, como su bajo costo y la gran cantidad de usuarios que pueden visualizarlos son un atractivo para captar clientes potenciales y fidelizar a los ya establecidos (Lin, K.-Y., \& Lu, H.-P., 2011). Es por esto, que se le debe dar gran importancia y énfasis a estos sitios a la hora de competir e innovar en el ámbito empresarial, ya que, esta herramienta puede contribuir en el posicionamiento y reconocimiento de una marca, producto o servicio, enfocándose principalmente en estrategias online.

Por lo tanto, los SNSs son un medio para interactuar directamente con los usuarios, quienes serán sus potenciales compradores. Estos pueden generar una relación de fidelidad cliente-empresa, incluso llegando a crearse comunidades de admiradores y defensores de la marca (Kietzmann, J.H et al., 2011).

Actualmente, es posible acceder a investigaciones de SNSs de posicionamiento y reconocimiento de la marca, sin embargo, la presente investigación se enfoca en determinar la utilidad en la venta de productos y servicios, a través de las estrategias empleadas en Facebook, lo cual es desconocido para la región de Coquimbo. Por lo tanto, es un estudio de gran utilidad para las empresas regionales que quieran manejar de manera eficiente los Sitios de Redes Sociales, potenciarlos, y que se refleje un crecimiento en estas, desde el punto de vista económico. Entonces, se puede decir que no existe otra investigación similar realizada previamente en la Región de Coquimbo que aborde este tema, por lo que esta propuesta es innovadora y se apega a la realidad de un mundo dominado por la tecnología y los SNSs.

Esta investigación se basa en el SNS de "Facebook", la plataforma social más usada por las empresas en estudio para interactuar con los usuarios. Esto se debe a que alrededor de un $70 \%$ de la población de nuestro país se considera un usuario activo de internet y casi el $90 \%$ de los jóvenes entre 15 y 29 años utilizan los SNSs.

El objetivo principal de este artículo es analizar la efectividad en el uso de Facebook para la venta de productos y/o servicios de empresas de la Región de Coquimbo. Debido a que es una herramienta que no ha sido explotada en su totalidad y que 
puede aportar en el aumento de las ventas de la empresa.

A continuación se procede de la siguiente manera. En la siguiente sección, se explican los conceptos claves a tratar en la investigación y se da a conocer las dos empresas consideradas para el estudio. Luego se presenta la metodología, basado en un esquema metodológico propuesto, continuando con los resultados, análisis y hallazgos obtenidos. Finalmente, se dan a conocer las conclusiones y propuestas de este articulo.

\section{MARCO TEÓRICO}

En un mundo cada vez más interconectado, las empresas deben estar atentas a las nuevas tendencias y desarrollo de tecnologías para no quedar atrás en sus intentos por lograr un mejor posicionamiento de marca y crecimiento de sus ventas, para lo cual deben utilizar todas las herramientas que estén a su alcance.

Existen dos conceptos claves para esta investigación: Sitios de Redes Sociales (SNSs por la sigla en inglés de social network sites) y Boca a Boca Electrónico (eWOM por la sigla en inglés de Electronic Word of Mouth). Estos dos conceptos son explicados a continuación.

\section{Sitios de Redes Sociales}

Según Boyd y Ellison (2007), son sitios Web en los cuales usuarios crean perfiles, ya sean públicos o con algunas reglas de privacidad, que les permiten interactuar con otros usuarios y a la vez navegar en sus perfiles. Es muy importante el correcto manejo de las redes sociales analizando a los clientes, ya que la comunidad de usuarios se siente copropietaria de la marca. Como consecuencia, los miembros desarrollan un sentido de responsabilidad, por lo que según su interpretación colectiva pueden llegar a promover diversas formas de boicot u oponerse a un crecimiento que consideren excesivo, al definir el éxito de la marca en términos distintos de los de la empresa (Muniz\&O'Guinn, 2001).

Los consumidores se están alejando de las fuentes tradicionales de publicidad: radio, televisión, revistas y periódicos, al mismo tiempo que demandan acceso inmediato a la información para su conveniencia (Rashtchy, 2007). Por lo cual, es de importancia identificar los SNSs que generan mayor influencia en las decisiones de los consumidores, tomando en cuenta las opiniones de los clientes sobre un producto o servicio de determinada empresa, en este caso se utiliza el SNS más importante en cuanto a cantidad de usuarios en el mundo: Facebook (eBizMBA, 2015). 


\section{Facebook}

Facebook (http://facebook.com), es un SNS, creado por Mark Zuckerberg, de carácter masivo. Este SNS puede alojar contenido en texto, imagen y video, por lo que la convierten en una plataforma atractiva para los usuarios, con pocas limitantes de extensión y tipo de contenido.

La comunicación se da en el "muro" de cada usuario y a través de mensajes internos que brindan mayor privacidad. Este SNS ofrece la posibilidad de crear páginas de seguidores, las que son utilizadas en su mayoría por empresas y de las cuales surgen las llamadas "comunidades de marca". Otra de las características de Facebook, es que ofrece servicios pagados donde una empresa puede publicar sus productos y generar estrategias de promoción.

\section{Boca a Boca Electrónico (eWOM)}

En Word of Mouth (WOM) o boca a boca, toda comunicación es oral y personal relacionada a una marca, producto o servicio, en el que el receptor del mensaje percibe la intención del emisor como una acción no comercial (Arndt, 1967). Los vendedores han reconocido la importancia del fenómeno del boca a boca, contextualizándolo como un fenómeno de origen natural, y que afecta a la mayoría de las decisiones de compra (Brooks, 1957).

Por lo tanto, es una fuente importante de obtención de información, ya que se obtiene de las comunicaciones interpersonales e influye significativamente en evaluaciones de productos y decisiones de compra (Mahajan et al., 1990). De hecho, el WOM ha demostrado ser más poderoso que la información impresa principalmente porque la información del boca a boca se considera más creíble (Borgida \& Nisbett, 1977).

Los medios sociales han amplificado el poder de las conversaciones de consumidor a consumidor en el mercado al permitir que una persona pueda comunicarse literalmente con cientos o miles de otros consumidores de forma rápida y con un esfuerzo relativamente pequeño, es lo que denominamos boca a boca electrónico (eWOM) (Chu, S.-C., \& Kim, Y., 2011). Este término se define como el acto de intercambiar información a través de Internet sobre un producto 0 servicio de una determinada organización (Grewal et al., 2003). Por lo tanto, debido a que los administradores no pueden controlar directamente las conversaciones sobre una determinada marca, deben utilizar métodos para influir y dar forma a estas discusiones de una manera que sea consistente con la misión y los objetivos de rendimiento de la organización (Mangold \&Faulds, 2009).

Según los autores Xu et al.(2012), actualmente la popularidad de los SNSs hace que cada vez más usuarios están contribuyendo con sus comentarios y opiniones en línea sobre productos, personas, organizaciones y entidades, los que a menudo tienen influencia directa en las decisiones de compra de los consumidores y las impresiones del público sobre las empresas. 


\section{Páginas de Marca}

Las empresas han comenzado a explorar la posibilidad de utilizar los SNSs como plataformas para llevar a cabo un marketing dirigido y una gestión de la reputación para los negocios en línea. Esto es debido al poder de influencia conjunta que un pequeño grupo de usuarios activos podría tener en un gran número de decisiones de compra de los consumidores y de la percepción del público sobre las capacidades de las empresas, debido a esto es que se crean las páginas de marca 0 "Brand Page". También se les conoce popularmente como "Fan Page".

Los medios de comunicación social constituyen excelentes vehículos para fomentar las relaciones de las empresas con los clientes (de Vries et al., 2012). Una forma específica de hacer esto es crear una página para seguidores en los SNS, donde las empresas pueden agregar mensajes y comentarios que contengan fotos, vídeos, cuestionarios o información sobre sus marcas. Los clientes pueden convertirse en seguidores de estas páginas de marca, y posteriormente indicar que les gusta el mensaje o comentario publicado. El número de seguidores, de "me gusta" y comentarios sobre los mensajes publicados por la empresa reflejará la popularidad que tiene la página de la marca.

\section{Comunidad de marca}

Desde el punto de vista de la empresa, la aparición de las comunidades de marca se puede entender como la última etapa del marketing relacional. Estas comunidades son grupos sociales que comparten un interés notable por el producto o servicio que ofrece una empresa, por lo cual la marca ocupa un lugar central en la vida de estas comunidades, siendo la lealtad una cuestión de gran importancia (García Ruiz, 2005).

La nueva forma de comunicación, a través de SNSs, se ve reflejada en la proliferación de las "Comunidades de Marca”, lo que Muniz, A., \& O'Guinn, T. (2001) definen como grupos sociales cuyos miembros comparten una gran lealtad a una determinada marca comercial.

\section{Estrategias en SNSs}

Hoy en día los SNSs son de gran importancia para el desarrollo de diversas empresas debido a que, los usuarios utilizan al menos un $22,5 \%$ de su tiempo en redes sociales como Twitter, Facebook y YouTube (Pring, 2012), por lo tanto, es una cifra ineludible para las empresas y la evolución de éstas.

Respecto a las empresas de turismo, los SNSs tienen un gran impacto en la comunicación de su marca e identidad, pero este impacto muchas veces no es aprovechado por parte de las empresas, debido a que existe escaso conocimiento sobre la influencia en la comunicación de la marca sobre un destino turístico (María Munar, 2011). 
En el ámbito estratégico de los SNSs se confirma que los contenidos generados por los usuarios o seguidores a través del SNS tienden a percibirse como una información más fiable que la ofrecida por otras fuentes más institucionales como las páginas webs (Leung et al., 2013).

Las empresas de turismo suelen desaprovechar las características potenciales que poseen las SNSs, como es la interacción y diálogo que se puede establecer con los seguidores, ya que, a través de las preguntas, que son una invitación directa al usuario a ofrecer una respuesta, se podría facilitar una mayor interacción (Huertas et al., 2015).

En el aspecto de tipo de publicación, existen publicaciones o estados, las que pueden incluir fotos 0 videos y enlaces a otras páginas o contenidos. Respecto a esto, las publicaciones que reciben mayor reacción por parte de los usuarios son los "post" con fotografías, luego los siguen en menor medida los videos, posteriormente los enlaces y por último los estados sin contenido adicional, que no generan casi ninguna reacción (Huertas et al., 2015).

\section{SNS en la región de Coquimbo}

No existen estudios o estadísticas que indiquen el porcentaje de empresas de la región de Coquimbo que utilizan las SNS para promocionar o interactuar con sus clientes, pero si lo llevamos a una aproximación de lo que sucede a nivel nacional podríamos estimar que al menos el $60 \%$ de las empresas posee un perfil en los SNS, y donde el de mayor aceptación es Facebook (Burson-Marsteller, 2010; Gobierno de Chile, 2015).

Un ejemplo de éxito en el uso de SNS en la región es el caso de la Municipalidad de La Serena, la que se ha convertido en una de las municipalidades con más seguidores en Facebook de Chile (Redesign republic, 2014). Gracias a la gestión del departamento de comunicaciones de la Municipalidad de La Serena que administra el sitio, se informando e interactuando con los usuarios, se ha creado una red que bordean los 25.000 seguidores. Todo esto ha generado un reconocimiento de parte de los usuarios de SNS, ya sean habitantes o no de la ciudad, los que valoran la apuesta comunicacional de la municipalidad serenense.

A continuación, se describen las empresas regionales que son parte de la investigación.

\section{Callegari Automotriz}

Callegari es una empresa de carácter familiar creada en 1969 por el inmigrante italiano Attilio Callegari Bresadola junto a su esposa Eligia Panizza Vareschi, quienes forjaron los cimientos para lo que actualmente es una de las empresas más reconocidas en la región de Coquimbo. 
Las principales actividades de Callegari Automotriz son la comercialización, financiamiento y respaldo técnico de vehículos motorizados, ya sean nuevos 0 usados. Debido a esto, la empresa hace esfuerzos por mantener una directriz enfocada a la excelencia de cada uno de los servicios entregados, contando con personal altamente capacitado y en línea con la filosofía de la empresa. Además, cuenta con infraestructura de primer nivel, tanto en los locales de ventas, como en los talleres de servicio técnico y de pintura, los que cuentan con maquinaria y herramientas de última generación, teniendo en cuenta la alta competitividad del mercado y el alto grado de conocimiento e información de los clientes.

En la actualidad, la empresa comercializa dieciocho marcas de vehículos: BYD, Chery, Chrysler, DFSK, Dodge, Fiat, Ford, Jeep, Kia, MG, Mitsubishi, Peugeot, RAM, SSangYong, Subaru, Tata, ZX Auto y Volvo, además de siete marcas de camiones: Ford, Higer, Hino, Hyundai, Jmc, Shacman e Iveco. Asimismo, cuenta con nueve sucursales en la ciudad de La Serena, seis en Copiapó, una en Coquimbo y una en Ovalle.

En cuanto a las plataformas online, destacan la página web de la empresa (www. callegari.cl), la cual ofrece a los visitantes información de los productos y servicios ofrecidos, así como los medios de contacto, y los SNSs, Facebook y Twitter, en los que la empresa posee un perfil con el que interactúa con los usuarios seguidores de su marca.

La página de la marca en Facebook, Callegari Automotriz, al 22 de Octubre de 2015 cuenta con 2,462 seguidores (Callegari Automotriz, 2015), Ios que comentan, generalmente de forma positiva para la empresa y dan "me gusta" constantemente a las publicaciones de la página, generando una relación de cordialidad con sus seguidores.

\section{Turismo Delfines}

Turismo Delfines, Tour Operador y Agencia de Viajes \& Transporte es una empresa dedicada al turismo emisivo y receptivo incluyendo el transporte privado de pasajeros. Fue creada en el año 2000, en la ciudad de La Serena, Región de Coquimbo.

Ofrece programas para recorrer los principales destinos turísticos de la región de Coquimbo, con turismo tradicional (Valle del Elqui, Valle del Limari, Valle del Encanto Isla Damas, Ruta Mistraliana, Desierto Florido, entre otros), Turismo Astronómico (Observatorio Mamalluca, Observatorio Collowara y Observatorio del Pangue). Además de otros destinos turísticos a nivel nacional e internacional, como tours por el caribe o países sudamericanos.

La empresa Turismo Delfines actualmente es uno de los principales Operadores Turísticos de la región de Coquimbo, debido a su política de calidad de servicio que 
la ha llevado a conseguir diversos reconocimientos otorgados por la Municipalidad de La Serena y por SERNATUR.

Turismo Delfines posee un sitio web (http:// www.turismodelfines.com), en donde se destacan en detalle los tour y programas turísticos ofrecidos, además de atractivas fotografías de estos. También posee perfiles en las SNSs, Facebook y Twitter en donde publican información sobre los destinos turísticos y promociones de estos.

Al día 25 de octubre de 2015 la página de Turismo Delfines en Facebook posee 2,211 seguidores (Turismo Delfines, 2015) y variadas opiniones y comentarios positivos respecto a los servicios prestados o sobre información que requieren quienes desean adquirir los servicios turísticos.

\section{METODOLOGÍA}

La Tabla 1, que se muestra a continuación, establece un resumen metodológico de los objetivos específicos con sus respectivas actividades e instrumentos utilizados en el estudio.

Tabla 1. Esquema Metodológico, Resumen metodológico.

\begin{tabular}{|l|l|l|}
\hline \multicolumn{1}{|c|}{ Objetivos específicos } & \multicolumn{1}{|c|}{ Actividades } & \multicolumn{1}{c|}{ Instrumento } \\
\hline $\begin{array}{l}\text { Reconocer las estrategias } \\
\text { de Facebook de las } \\
\text { empresas regionales }\end{array}$ & $\begin{array}{l}\text { Reunion con los encargados } \\
\text { de SNSs de Turismo Delfines } \\
\text { y Callegari Automotriz }\end{array}$ & $\begin{array}{l}\text { Entrevistas con encargados } \\
\text { de SNSs }\end{array}$ \\
\hline $\begin{array}{l}\text { Comparar las Fan Page de } \\
\text { las empresas regionales }\end{array}$ & $\begin{array}{l}\text { Tabular y analizar los datos } \\
\text { obtenidos en las encuestas. } \\
\text { Comparación de resultados }\end{array}$ & $\begin{array}{l}\text { Encuestas a seguidores de } \\
\text { Facebook }\end{array}$ \\
\hline $\begin{array}{l}\text { Identificar las compras } \\
\text { influenciadas por las Fan } \\
\text { Pages }\end{array}$ & $\begin{array}{l}\text { Estudio y analisis de } \\
\text { resultados. } \\
\text { Comparación de resultados }\end{array}$ & $\begin{array}{l}\text { Utilización de información } \\
\text { recopilada de objetivos } \\
\text { anteriores }\end{array}$ \\
\hline
\end{tabular}

\section{Modelo Metodológico}

Para que la investigación tenga un enfoque correcto, en necesario que tenga un carácter cuantitativo, basado en resultados numéricos obtenidos a través de encuestas a un grupo de personas que conforman el tamaño de muestra, y así responder las interrogantes planteadas en la investigación. Se aplica este modelo metodológico por la facilidad de ordenar y utilizar los datos obtenidos de forma estadística.

Además, se plantea un test de hipótesis con el fin de determinar si la compra de productos 0 servicios de parte de los seguidores es influenciada de alguna manera por los contenidos publicado por la empresa en su Fan Page. 


\section{Tamaño de muestra}

En la Imagen 1 se establece la fórmula utilizada para calcular la muestra para la población, la cual corresponde a los seguidores de Facebook de las empresas "Callegari Automotriz" y "Turismo Delfines" al día 1 de octubre del 2015.

Figura 1: Fórmula de tamaño de muestra para población finita

$$
n=\frac{K^{2} * p^{*} q^{*} N}{(N-1) * e^{2}+K^{2} * p^{*} q}
$$

Fuente: Apuntes de investigación de mercado, Andrés Araya

Dónde:

- $\mathrm{n}=$ tamaño de la muestra.

- $\mathrm{k}=$ constante que depende del nivel de confianza prefijado.

- $\quad p=$ porcentaje de la población que posee la característica para este estudio. Lo cual correspondería a la población que es influenciada por las redes sociales.

- $q=$ porcentaje de la población que no posee la característica estudiada (1-p).

- $\mathrm{N}=$ tamaño del universo 0 tamaño de la población.

- $\mathrm{e}=$ error absoluto.

El estudio utiliza los parámetros con un $95 \%$ de confiabilidad, por lo tanto, se asume la constante $K$ con un valor de 1.96, el porcentaje de error absoluto es de $10 \%$, para los parámetros $q$ y $p$, al no conocerse este último, se asume máxima incertidumbre, por lo cual $p=q=50 \%$. Debido a que se estudian dos empresas con distintos números de seguidores, se determinarán el tamaño de muestra respectivo para cada uno. Dado lo anterior, la empresa "Callegari Automotriz", que posee 2.195 seguidores, arroja un resultado de 95,68\% como tamaño de muestra a encuestar, lo que debe aproximarse a 96 seguidores, en tanto "Turismo Delfines", que posee 2.060 seguidores, arroja un resultado de 95,41 como tamaño de muestra, por lo que se hace necesario encuestar a 96 seguidores. La elección de la muestra se realiza al azar, por lo tanto, todos los seguidores tienen la misma posibilidad de responder, ya que se publica en las "Fan Page" de las empresas invitándolos a responder un breve cuestionario.

\section{Método de recolección de datos}

Para el cumplimiento de los objetivos fue necesario realizar una serie de actividades, entre las cuales se destacan la entrevista y encuestas. Entrevistas personales 
semiestructurada, con preguntas preparadas y posibilidad de realizar contra preguntas. Este cuestionario se aplica a cada encargado o Community Manager del SNSs de Facebook de "Turismo Delfines" y "Callegari Automotriz".

Encuestas, con preguntas de tipo dicotómica, selección múltiple y Likert. Este instrumento se aplica a la población objetivo, los seguidores de las Fan Page de "Turismo Delfines" y "Callegari Automotriz", para detectar si se logra la compra del producto/servicio por parte del seguidor y si se utiliza la Fan Page en algún aspecto de su compra. El diseño de la encuesta online se lleva a cabo con la aplicación de formularios de Google Docs. . Esta encuesta se difunde en las Fan Page de cada empresa, por parte de los Community Manager para asegurar la respuesta de los seguidores de las respectivas páginas de marca.

\section{RESULTADOS}

\section{Reconocer las estrategias de SNS}

Para detectar las principales estrategias en el Fan Page de Facebook que utilizan "Callegari Automotriz" y "Turismo Delfines" para concretar la venta de sus productos/servicios, se realiza una entrevista a Marcelo Rivera (Gerente de Marketing) y Luis Pérez (Ejecutivo de Ventas) respectivamente. A continuación, se describe las principales estrategias de las empresas.

\section{Callegari Automotriz}

Alineamiento con Marketing: "Callegari Automotriz" alinea todas sus redes a partir del plan de marketing que se realiza, es decir, si en un mes se realizan campañas de publicidad para la venta de un tipo de vehículo específico, todos sus SNSs apoyan esta campaña publicando fotos y características del vehículo.

Noticias del rubro: En la Fan Page de "Callegari Automotrz" se publican contenidos: noticias, fotos, videos e información relacionados al sector automotriz, especialmente de nuevos modelos de vehículos que saldrán al mercado.

Información sobre promociones y sucursales: El encargado de la Fan Page de "Callegari Automotriz" publica constantemente contenido relacionado a las formas de financiamiento, bonos, tipos de vehículos y además la ubicación y horario de las sucursales, con lo cual los seguidores podrán encontrar el lugar y el momento adecuado para visitar físicamente dependencias de la empresa. 
La empresa no utiliza eficientemente la Fan Page, debido a que no creen que sea un aporte en sus ventas y desaprovechan beneficios que otorga este SNS. Se utiliza Facebook para estar acorde a la actualidad empresarial y tener su Fan Page al igual que las demás empresas. Puede que sus publicaciones sean bastante escasas, pero se rescata el hecho de que logran informar al seguidor con sus productos e interactuar con ellos.

\section{Turismo Delfines}

Lo más alcanzado: A través de esta estrategia "Turismo Delfines" se basan en analizar qué tipo de turismo es el más atrayentes para los seguidores, este análisis se logra por medio de las estadísticas que entrega Facebook de lo más alcanzado (visto).

Botón contactarse: Otra estrategia que se utiliza son las opciones (botones) que entregan las Fan Page, como: reservar, comprar registrarse usar aplicación, entre otras. Donde "Turismo Delfines" utiliza el botón de "Contactarnos", que dirige al seguidor aun formulario de su página web (sumando visitas), que los direcciona al correo de ellos. Algunos extranjeros llegan en primera instancia a la empresa por medio de Facebook y pulsan ese botón que los contacta a su página web y correo.

Respuesta a tiempo: "Turismo Delfines" tiene como margen máximo de respuestas a los comentarios y preguntas por inbox (mensaje interno) a sus seguidores e interesados de 20 minutos dentro del horario de atención de lunes a viernes (10:00 a 19:00 hrs).

Ventas en temporada baja: Esta estrategia utiliza Facebook para aumentar sus ventas en temporada baja, debido a que ofrecen a través de las publicaciones en Facebook lo que no han vendido por otros medios para completar sus cupos de venta.

Fin de Semanas Largos: La empresa promociona sus tours con mayor frecuencia para los días previos a fin de semanas largos., a través de las publicaciones en su Fan Page. La empresa no ha realizado un buen uso de la Fan Page. Debido a que estas estrategias no se alinean directamente con los seguidores. Es decir, no existe un plan estratégico estudiado y planteado, se realizan estrategias temporales según diversos factores. Aunque el formato y las publicaciones son correctas, falta mayor énfasis en lo planteado anteriormente.

\section{Comparación de resultados del estudio de las Fan Page de las empresas regionales}

Las comparaciones de las empresas se hacen necesaria debido a las diferencias que puedan ofrecer al no pertenecer al mismo rubro, ofreciendo por un lado la 
empresa "Callegari Automotriz" la venta de automóviles (producto duradero) y por el otro, "turismo Delfines" ofreciendo la venta de tours (servicio), ya que la caracterización de los seguidores es distinta. Mientras en la empresa de turismo es posible que los seguidores de la empresa sean no sólo de la región de Coquimbo, para Callegari, la posibilidad de que los seguidores residan en otra región o incluso en el extranjero es mucho menor.

Los resultados obtenidos indican que al menos el $49 \%$ de los seguidores de "Turismo Delfines" ha ingresado y se ha informado sobre sus servicios, mientras en "Callegari Automotriz" corresponde al 52\%, por lo que se observa una similitud en los resultados en este aspecto, esto quiere decir que gran parte de los seguidores al menos han observado el contenido de la Fan Page y han obtenido mayor información sobre lo que ofrecen estas empresas, por lo tanto es posible que ante una necesidad de adquirir algún producto 0 servicio que ofrecen las empresas mencionadas, serán a lo menos consideradas por los seguidores. También se destaca que en ambas empresas el porcentaje de seguidores que ha llegado a hacer consultas luego de haberse informado sobre lo que ofrecen en la Fan Page es de alrededor de un $13 \%$.

También se logra observar que al menos el $10 \%$ de los seguidores ha adquirido algún servicio de "Turismo Delfines", mientras que el $24 \%$ de los seguidores ha adquirido algún producto o servicio de la empresa "Callegari Automotriz". Por lo tanto, son los seguidores de "Callegari Automotriz" quienes han comprado en un mayor porcentaje respecto a "Turismo Delfines". Esto se debe a que los seguidores de la empresa "Callegari Automotriz" tienen una decisión previa de adquirir el producto o servicio, y que la información provista por la Fan Page es un apoyo para concretarla, además la empresa "Callegari Automotriz" realiza una mayor interacción con sus seguidores logrando generar mayor cercanía.

Otro aspecto a considerar es la influencia de la Fan page en la decisión de compra de los seguidores. Se concluye que solo un $5 \%$ de los seguidores de "Turismo Delfines" considera que la Fan Page ha influido en la contratación de algún servicio de la empresa. También, se muestra que un 15\% de los seguidores de "Callegari Automotriz" consideran que la Fan Page ha influido en algún aspecto de su compra. Esto se puede explicar porque los seguidores de "Callegari Automotriz" tienen una decisión tomada previamente sobre adquirir el producto (Vehículo) que ofrecen, por lo tanto, sólo busca informarse mejor para encontrar el momento adecuado para concretar esta compra, mientras que, para el servicio de turismo, es más común que los seguidores observen la Fan Page para planear adquirir el servicio en un plazo futuro, sin tener una decisión tomada.

Los usuarios de Facebook son seguidores de las Fan Page siendo el motivo principal en "Turismo Delfines" el interés en la información, fotos y videos que comparte, seguido por el interés en los servicios que ofrece, lo que en su conjunto suma el $60 \%$ de las preferencias. Mientras que en el caso de "Callegari Automotriz" estos motivos representan en torno a un $61 \%$ siendo el interés en los productos que ofrece, el principal motivo. 
Por lo tanto, es de gran importancia ofrecer a los seguidores un contenido apropiado, con noticias e información importante relacionada al servicio ofrecido, generando un interés en los seguidores por acceder a la Fan Page y luego adquirir los productos y servicios ofrecidos.

Para los seguidores de ambas empresas es interesante la información, fotos y videos compartidos por la empresa, seguido por los concursos, beneficios y promociones que realizan estas. Por lo tanto, al momento de crear un plan estratégico no se diferenciaría al aplicarlo en empresas con ventas de distinta naturaleza, sólo hay que tener consideración en la información a proveer, ya que debe estar relacionada al rubro de la empresa.

En cuanto a la información específica que prefiere el seguidor de "Turismo Delfines", se logra dilucidar que tiene relación con el tipo de turismo astronómico y por destilerías y viñedos, lo que indica que los seguidores de la empresa, en su mayoría de la misma región, prefieren admirar los destinos que más representan a la región de Coquimbo, generándose una identificación con los atractivos turísticos locales.

En el caso de los seguidores de "Callegari Automotriz", la información que más atrae a los seguidores es la respectiva a beneficios y promociones que pudiera entregar la empresa, representando el $73 \%$ del total, por lo tanto, es necesario mantener actualizada la Fan Page con este tipo de información, de modo que los potenciales clientes puedan concretar la compra al ver los beneficios que ofrece la empresa.

Este aspecto es importante, ya que, en la actualidad, los clientes evalúan una serie de antecedentes antes de tomar una decisión que implique realizar un gasto elevado, como lo es la compra de un vehículo o la mantención de este, entonces una adecuada promoción o beneficios que destaque por sobre otras empresas, les dará una ventaja competitiva en el mercado.

\section{Identificar la influencia de las Fan Page}

Para identificar la influencia de las Fan Page en la venta de productos/servicios, en primera instancia se realiza un test de hipótesis con los datos obtenidos de la encuesta para corroborar que los resultados son significantes, posteriormente se realiza análisis de datos cruzados y luego el reconocimiento de las variables que más influyen en el estudio, con lo cual se pretende descubrir los motivos por los cuales los seguidores visitan la Fan Page y la información que más le atrae. 


\section{Test de Hipótesis}

Se propone, en conjunto con las empresas de estudio, que con un $40 \%$ de respuestas positivas de que los seguidores han comprado el producto y han sido influenciados en algún aspecto por la Fan Page de la empresa, se considera que esta plataforma es de utilidad para promocionar los servicios entregados y atraer nuevos clientes en la región de Coquimbo, por lo tanto:

Hipótesis Nula: No se cumple lo que se plantea, que al menos un $40 \%$ de las personas que adquirieron el producto o servicio fueron influenciados por la Fan Page.

Hipótesis alternativa: Al menos un $40 \%$ de los seguidores adquirió el producto 0 servicio influenciado por la Fan Page.

$$
\begin{aligned}
& H_{0}=P<0.40 \\
& H_{1}=P \geq 0.40
\end{aligned}
$$

*Donde $\mathrm{P}$ es la proporción de personas que adquirió el producto 0 servicio influenciados por aspectos de la Fan Page.

Porcentaje de confianza del test: $95 \%$

Nivel de significancia: $\alpha=0.05$ por lo tanto su valor $Z$ tabla será de 1.645

Ecuación 1:Error estándar de la proporción especificada en la hipótesis nula:

$$
S_{p}=\sqrt{\frac{P(1-P)}{n}}
$$

Donde:

$\mathrm{P}=$ proporción especificada en la hipótesis nula

$\mathrm{n}=$ tamaño de la muestra

Por lo tanto, según los datos provistos anteriormente:

$$
S_{p}=\sqrt{\frac{0.40(1-0.40)}{100}}=\mathbf{0 . 0 4 9}
$$


Ecuación 2: Cálculo del estadístico de prueba "Turismo Delfines":

$$
\begin{gathered}
z=\frac{\text { (proporción observada }- \text { proporción hipotesis nula) }}{S_{\mathrm{p}}} \\
z=\frac{0.50-0.40}{0.049}=2.0408
\end{gathered}
$$

Ecuación 3: Cálculo del estadístico de prueba "Callegari Automotriz":

$$
\begin{gathered}
z=\frac{\text { (proporción observada }- \text { proporción hipotesis nula) }}{S_{\mathrm{p}}} \\
z=\frac{0.609-0.40}{0.049}=\mathbf{4 . 2 6 5 3}
\end{gathered}
$$

Donde, para ecuación 2 y 3 :

Proporción observada: es la proporción de personas que adquirió el producto 0 servicio influenciados por aspectos de la Fan Page de cada empresa.

Proporción hipótesis nula: es la proporción esperada, de que al menos un 40\% de las personas que adquirieron el producto o servicio fueron influenciados por la Fan Page.

$S_{p}$ : Error estándar de la proporción especificada en la hipótesis nula.

Como se aprecia en los resultados, en ambos casos la hipótesis nula se rechaza porque los valores de la Z calculados, siendo 2.0408 para "Turismo Delfines" y 4.2653 para "Callegari Automotriz" resultan mayores que el valor crítico $Z$ de una tabla de distribución normal (1.645) con un intervalo de confianza del $95 \%$. Por lo tanto, se demuestra que el test indica que es significativo, con un 95 por ciento de confianza ( $1-\alpha=0.95)$, entonces, si al menos el 40 por ciento de los seguidores, que adquieren un producto 0 servicio de las empresas se ven influenciados en algún aspecto por la Fan Page de la empresa, esta compra resultará significativa para la empresa.

Análisis de datos cruzados de la empresa "Turismo Delfines".

Luego del análisis se puede apreciar que al menos el 10\% de quienes han realizado consultas a la empresa están motivados por el interés en la información, fotos y videos que se han publicado en la Fan Page de "Turismo Delfines". Además, quienes finalmente han comprado luego de ser seguidores, lo han hecho principalmente 
motivados por un interés en el servicio ofrecido. En el total de la muestra, quienes han mirado y se han informado sobre los servicios ofrecidos representan el 49\%, mientras que los que además han consultado en la empresa representan el 12\%, finalmente quienes indican que luego de consultar han comprado representan el $2,1 \%$.

Se determina la relación Motivo del usuario de ser seguidor de la Fan Page v/s Uso de la Fan Page por parte del seguidor. Esto nos indica que, si bien la Fan Page puede tener influencia en la decisión de compra, generalmente se requiere una decisión previa del seguidor a querer adquirir el servicio, ya que no todos consultan directamente a la empresa antes de comprar. La segunda relación obtenida es Compra del servicio por el seguidor v/s Influencia de Fan Page en la compra del servicio. Se pretende medir la influencia de la Fan Page en quienes han adquirido algún producto o servicio de la empresa. Al observar el cruce de variables entre la compra y la influencia de la Fan Page en la compra, lo que nos da como resultado que el $50 \%$ de quienes han contratado servicios de la empresa 10 ha hecho influenciado en algún aspecto por la Fan Page mientras el restante $50 \%$ no ha sido influenciado por la Fan Page. Si se analiza en el total de la muestra, está influencia representa solo un 5,2\%. Por lo tanto, se hace necesario mejorar este porcentaje, logrando que un número mayor de personas que se hacen seguidores de "Turismo Delfines" concrete una compra gracias a la ayuda de esta plataforma.

\section{Análisis de datos cruzados de la empresa "Callegari Automotriz"}

Con respecto al Motivo del usuario de ser seguidor de la Fan Page v/s Uso de la Fan Page por parte del seguidor. El total de quienes han comprado lo han hecho por interés en los servicios ofrecidos por la empresa, mientras que la mayor parte de quienes han consultado a la empresa lo han hecho por el interés en la información fotos y videos de la empresa, lo que representa un 10,4\% del total de los seguidores. Si tomamos en cuenta el total de seguidores, sólo un $12,5 \%$ ha consultado en la empresa y un 6,3\% finalmente ha comprado.

Por lo tanto, al igual que en la empresa de turismo, es necesario generar estrategias para lograr que los clientes que aún dudan de adquirir los productos y servicios de la empresa logren concretar la compra, a través de un marketing que incentive un apego de los seguidores por la empresa, los cuales lleguen a preferir a "Callegari Automotriz" por sobre la competencia.

Respecto a la Compra del producto por el seguidor v/s Influencia de Fan Page en la compra del producto. Se aprecia que al menos el $60 \%$ de quienes han adquirido algún producto o servicio de "Callegari Automotriz" lo han hecho influenciado en algún aspecto por su Fan Page, mientras un 39,1\% no ha sido influenciado. En tanto, tomando en consideración el total de seguidores, sólo el 14,6\% ha comprado influenciado por la Fan Page. Es decir, "Callegari Automotriz" tiene mayor llegada con aquellos clientes que finalmente deciden adquirir sus productos y servicios en 
comparación a "Turismo Delfines", sin embargo, este porcentaje aún es pequeño en comparación a la cantidad de seguidores.

\section{Confianza del seguidor en la Fan Page}

Los resultados obtenidos por la encuesta, el 84, 3\% de los seguidores de "Turismo Delfines" y un $86,5 \%$ de los seguidores de "Callegari Automotriz" considera que la información que entrega la empresa por la Fan Page es fidedigna. Mientras que un $69,8 \%$ de los seguidores de "Turismo Delfines" y un $73 \%$ de los seguidores de "Callegari Automotriz", considera que los comentarios que realizan otros seguidores son fidedignos. Y por último el 71,9\% de los seguidores de "Turismo Delfines" y un 69,8\% considera que las empresas responden honestamente con las dudas y reclamos. Por lo tanto, los usuarios que siguen la Fan Page de cada empresa en su mayoría confían en el contenido que se comparte en el SNS.

Es importante que se saque el mayor provecho a estos atributos que tienen las empresas, consolidando el lazo entre los seguidores y empresa para generar una mayor lealtad que con el tiempo se traduce en generar una empresa confiable y valorada por la sociedad como un aporte más allá del giro comercial.

\section{Influencia de la Fan Page en la compra}

El 79,2\% de los seguidores de "Turismo Delfines" y el 78,2\% de los seguidores de "Callegari Automotriz" considera que en las Fan Page de las empresas se mencionan aspectos importantes que consideraran a la hora de comprar: Mientras el 73, 9\%de los seguidores de "Turismo Delfines" y un $69,8 \%$ de los seguidores de "Callegari Automotriz" considera que los comentarios y calificación de los seguidores son útiles para tomar una decisión de compra. Y por último el 73,9\% de los seguidores de "Turismo Delfines" y un 74\% de los seguidores de "Callegari Automotriz" considera que la Fan Page de las empresas es un medio útil para comprar.

Tras evaluar estos aspectos se puede observar que los seguidores les es útil la Fan Page para tomar una decisión de compra, ya sea por la información que se comparte y las opiniones que entregan los seguidores que han comprado el producto/servicio.

\section{CONCLUSIÓN}

Claramente los SNSs, como Facebook, son de gran importancia en la actualidad, ya que poseen ciertas características que permiten, por ejemplo, llegar a una gran cantidad de público en tan sólo unos segundos. Por lo tanto, podemos decir que esta plataforma posee un gran valor para las empresas, ya que puede significar una oportunidad de darse a conocer, consolidarse o bien aumentar sus ventas. 
Como se puede observar en esta investigación, en la región de Coquimbo no existe un estudio que indique la influencia de los SNS, en específico de Facebook para la compra de productos o servicios, por lo cual el contenido provisto puede ser de utilidad para aquellas empresas que mantienen perfiles vigentes o bien deseen crearlos y que no tienen conocimiento de las estrategias que pueden utilizar y el efecto que puede producir en sus seguidores y potenciales clientes.

Según este estudio, que da a conocer la situación actual de esta plataforma social, los conceptos más importantes y estrategias de uso, y que incluye la realización de un test de hipótesis y un análisis estadístico de los factores de influencia en los seguidores, Facebook si influye en la decisión de comprar un producto 0 servicio en empresas regionales. Al mismo tiempo se determinó que las empresas regionales no utilizan efectivamente este SNS, ya que, desaprovechan los beneficios y características potenciales de Facebook para captar más seguidores y posibles clientes, debido a que no dirigen sus publicaciones al interés que tienen sus seguidores actuales.

A través de este estudio se logró identificar que la mayor parte de los seguidores son personas de un rango de edad de 18 a 35 años, con interés definidos a los cuales las empresas se pueden enfocar para generar un aumento en las ventas de sus productos/servicios y un reconocimiento de su marca.

Debido a lo anterior, se sugiere una serie de estrategias para utilizar de mejor forma la Fan Page, donde la más recomendable es interactuar con el seguidor constantemente, ya que al realizar esta acción, aumenta la fidelidad y compromiso, generando un eWom positivo por parte de sus seguidores a otros usuarios, lo que podría generar la formación de una comunidad de marca, la que representa la última etapa del marketing relacional.

Por lo tanto, las propuestas sugeridas para las empresas de la región de Coquimbo que deseen mejorar su efectividad de promoción y venta en Facebook son:

- $\quad$ Definir un nombre de usuario

- $\quad$ Definir un público objetivo

- $\quad$ Promocionar su Fan Page y publicaciones

- $\quad$ Lograr un eWom positivo

- $\quad$ Incentivar la creación de una "Comunidad de marca"

Finalmente, se determina que debe haber una correlación entre los objetivos de la empresa y las estrategias que utilizan en sus SNS, ya que, debe estar clara la dirección que pretende tomar la empresa con respecto a estas plataformas, para así administrarlas eficientemente y lograr atraer nuevos potenciales clientes. 


\section{REFERENCIAS}

ARNDT, J. (1967). Word of mouth advertising. [Advertising Research Foundation].

BOYD, D., \& ELLISON, N. (2007). Social Network Sites: Definition, History, and Scholarship. Journal Of Computer-Mediated Communication, 13(1), 210230. doi: 10.1111/j.1083-6101.2007.00393.x

BORGIDA, E., \& NISBETT, R. (1977). The Differential Impact of Abstract vs. Concrete Information on Decisions1. Journal Of Applied Social Psychology, 7(3), 258-271. https://doi.org/10.1111/j.1559-1816.1977. tb00750.x

BR00KS, R. (1957). "Word-of-Mouth" Advertising in Selling New Products. Journal of Marketing, 22(2), 154. https://doi. org/10.2307/1247212

BURSON-MARSTELLER. (2010). Latin America Social Media Check-Up. Slideshare.net. Retrieved 2010, from https://www.slideshare.net/ BMGlobalNews/latin-america-social-media-checkup?from=ss_embed

CALLEGARI AUTOMOTRIZ. (2015). Callegari Automotriz. Facebook.com. Retrieved 22 October 2015, from https://www.facebook.com/pages/ Callegari-Automotriz/212883872238572

CHU, S.-C., \& KIM, Y. (2011). Determinants of consumer engagement in electronic Word-Of-Mouth (eWOM) in social networking sites. International Journal of Advertising, 30 (1), 47-75. doi: 10.2501/IJA-30-1-047-075

eBizMBA. (2015). Top 15 Best Social Networking Sites and APPs | February 2020. Ebizmba.com. Retrieved 29 June 2015, from http://www.ebizmba. com/articles/social-networking-websites

GARCÍA RUIZ, P. (2005). Comunidades de marca. El consumo como relación social (42 vol., pp. 257-272). Ediciones Complutense.

GOBIERNO DE CHILE (2015). Plataformas sociales más utilizadas en las Instituciones Públicas. Guía Digital. Disponible en: http://www.guiadigital. gob.cl/articulo/plataformas-sociales-mas-utilizadas-en-las-institucionespublicas

GREWAL, R., CLINE, T., \& DAVIES, A. (2003). Early-Entrant Advantage, Wordof-Mouth Communication, Brand Similarity, and the Consumer DecisionMaking Process. Journal Of Consumer Psychology, 13(3), 187-197. https://doi.org/10.1207/s15327663jcp1303_01

HANNA, R., ROHM, A., \& CRITTENDEN, V.L. (2011). We're all connected: The 
power of the social media ecosystem. Business Horizons, 54 (3), 265273. doi:10.1016/j.bushor.2011.01.007

HUERTAS, A., SETÓ-PÀMIES, D., \& MÍGUEZ-GONZÁLEZ, M. (2015). Comunicación de destinos turísticos a través de los medios sociales. El Profesional De La Información, 24(1), 15-21. https://doi.org/10.3145/ epi.2015.ene.02

INSTITUTO NACIONAL DE ESTADÍSTICA. Informe demográfico 2014. Disponible en: http://www.ine.cl/canales/chile_estadistico/demografia_y_ vitales/proyecciones2014/proyecciones-de-poblacion-2014.xlsx

KIETZMANN, J.H., HERMKENS, K., MCCARTHY, I.P., \& SILVESTRE, B.S. (2011). Social media? Get serious! Understanding the functional building blocks of social media. Business Horizons, 54 (3), 241-251. doi: 10.1016/j. bushor.2011.01.005

LEUNG, D., LAW, R., VAN HOOF, H., \& BUHALIS, D. (2013). Social Media in Tourism and Hospitality: A Literature Review. Journal Of Travel \& Tourism Marketing, 30(1-2), 3-22. https://doi.org/10.1080/10548408.2013.7509 19

LIN, K.-Y., \& LU, H.-P. (2011). Why people use social networking sites: An empirical study integrating network externalities and motivation theory. Computers in Human Behavior, 27 (3), 1152-1161. doi: 10.1016/j. chb.2010.12.009.

PRING, C. (2012). Social media and internet statistics. The social skinny. Retrieved from http://thesocialskinny.com/216-social-media-andinternetstatistics-september-2012

MAHAJAN, V., MULLER, E., \& BASS, F. (1990). New Product Diffusion Models in Marketing: A Review and Directions for Research. Journal Of Marketing, 54(1), 1-26. https://doi.org/10.2307/1252170

MANGOLD, W., \& FAULDS, D. (2009). Social media: The new hybrid element of the promotion mix. Business Horizons, 52(4), 357-365. https://doi. org/10.1016/j.bushor.2009.03.002

MARÍA MUNAR, A. (2011). Tourist-created content: rethinking destination branding. International Journal Of Culture, Tourism And Hospitality Research, 5(3), 291-305. https://doi.org/10.1108/17506181111156989

MUNIZ, A., \& O'GUINN, T. (2001). Brand Community. Journal Of Consumer Research, 27(4), 412-432. https://doi.org/10.1086/319618

RASHTCHY, S. (2007). The user revolution. PiperJaffray, Investment Research. 
REDESIGN REPUBLIC. (2014). La Serena, un ejemplo de Branding. Redesignrepublic.com. Retrieved 29 June 2015, from http://www. redesignrepublic.com/2014/05/la-serena-un-ejemplo-de-branding/

DE VRIES, L., GENSLER, S., \& LEEFLANG, P. (2012). Popularity of Brand Posts on Brand Fan Pages: An Investigation of the Effects of Social Media Marketing. Journal Of Interactive Marketing, 26(2), 83-91. https://doi. org/10.1016/j.intmar.2012.01.003

TURISMO DELFINES. (2015). Turismo Delfines - Tour Operador \& Agencia de Viajes. Facebook.com. Retrieved 25 October 2015, from https:// www.facebook.com/Turismo-Delfines-Tour-Operador-Agencia-deViajes-127282913122

XU, K., GUO, X., LI, J., LAU, R., \& LIAO, S. (2012). Discovering target groups in social networking sites: An effective method for maximizing joint influential power. Electronic Commerce Research And Applications, 11(4), 318334. https://doi.org/10.1016/j.elerap.2012.01.002 